in

A STOCHASTIC MODEL OF THE GALACTIC MAGNETIC FIELD

Thomas B. Kaiser

Technical Report Number 73-034

September 1972

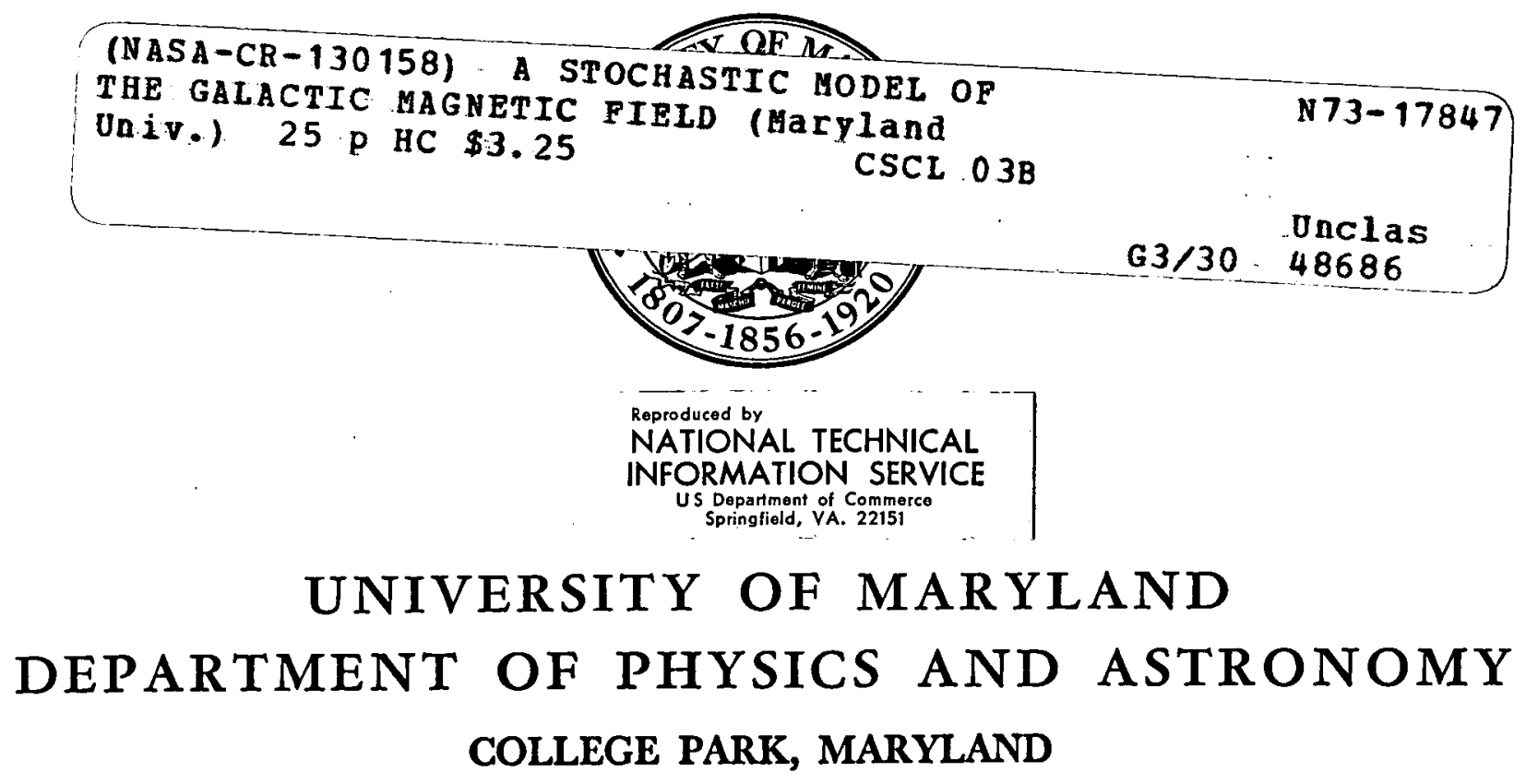




\title{
A STOCHASTIC MODEL OF THE GALACTIC MAGNETIC FIELD
}

\author{
THOMAS B. KAISER * \\ Department of Physics and Astronomy, University of Maryland \\ Received
}

\begin{abstract}
Existing stochastic models of the galactic magnetic field are considered and found to suffer certain defects. A new model is proposed which overcomes faults of the previous theories while retaining their strengths.
\end{abstract}

${ }^{*}$ This research was supported by the National Aeronautics and Space Administration under Grant NASA NGL 21-002-033. 


\section{INTRODUCTION}

A random component of the galactic magnetic field was first proposed by Fermi (1949) in order to explain the isotropy of cosmic rays. This possibility was considered in much greater detail by Jokipii and Parker (1969a, b), who showed that it offers a plausible mechanism for the escape of cosmic rays from the Galaxy in the relatively short $10^{6}$ years implied by observations of abundances of light cosmic ray nuclei. The model was given further credibility by Jokipii, Lerche and Schommer (1969), who showed that it is consistent with observed fluctuations in the polarization of starlight, and by Jokipii and Lerche (1969), who demonstrated a similar consistency in the case of Faraday rotation of pulsar signals. Jones (1971a) refined the treatment of Jokipii and Parker and derived results directly applicable to the determination of the statistical properties of cosmic ray parameters.

The purpose of the present paper is to consider a further refinement of the previous discussions which retains their strengths and overcomes some of their weaknesses. In §II a brief summary of the theories of Jokipii and Parker and of Jones is given. In $\S I I I$ an alternative mode1 is described and worked out in detail, and in $\S I V$ it is compared with observations. $\S \mathrm{V}$ presents conclusions and suggestions for further development. 


\section{EXISTING THEORIES}

Jokipii and Parker (1969a, b) point out that the velocity field of the interstellar gas is turbulent and random. Since the electrical conductivity is very high, the magnetic field is frozen into the gas motion, and hence is itself random. On the assumption of homogeneous velocity turbulence they are thus led to describe the magnetic field as a homogeneous stochastic process ${ }^{1}$. The total field is the sum of the average field, $\vec{B}_{0}$, assumed to lie along the local spiral arm, and a fluctuating field, $\vec{B}_{1}$, of much smaller magnitude. In a coordinate system with the $x$-axis along the arm and the z-axis perpendicular to the galactic plane,

$$
\vec{B}=B_{0} \vec{I}_{x}+\vec{B}_{1}
$$

Neglecting all terms of second order in the ratio $\left|\vec{B}_{1}\right| / B_{0}$, as well as spatial derivatives of $\vec{B}_{1}$ perpendicular to $\vec{B}_{0}$, the equations of a line of force passing through the origin are

$$
\begin{aligned}
& y(x)=\int_{0}^{x} \frac{\left[\vec{I}_{y} \cdot \vec{B}_{1}\left(\vec{x}^{\prime}\right)\right]_{y^{\prime}=z^{\prime}=0}}{B_{0}} d x^{\prime}, \\
& z(x)=\int_{0}^{x\left[\vec{I}_{z} \cdot \vec{B}_{I}\left(\vec{x}^{\prime}\right)\right] y^{\prime}=z^{\prime}=0} d_{0}^{\prime}
\end{aligned}
$$


For distances along the mean field much greater than the correlation length of the random field, L, the mean square displacement of a field line from the $x$-axis can be derived from eqs. (2):

$$
\left\langle y(x)^{2}\right\rangle \simeq\left\langle z(x)^{2}\right\rangle \simeq 1 / 2 \mathrm{Lx}
$$

Regarding the escape of cosmic rays from the Galaxy, this is the principal result obtained by Jokipii and Parker, because it shows that a field line wanders in a direction perpendicular to the mean field an arbitrarily large amount for increasing displacements along the average field. Therefore, any given field line eventually migrates to the surface of the galactic disk, allowing the cosmic rays trapped on it to escape (Jokipii and Parker 1969 b).

The major shortcomings of this treatment are the following: 1) It assumes that $\left|\vec{B}_{1}\right| \ll\left|\vec{B}_{0}\right|$, which certainly does not appear to be valid for the magnetic field of the Galaxy (Serkowski 1962; Jokipii and Lerche 1969);

2) Eq. (3) can be expected to hold only for $\left\langle z(x)^{2}\right\rangle \lesssim z_{D}^{2}$, where $z_{D}$ is the half-thickness of the galactic disk. This is because the actual field of the Galaxy is confined to the disk (Parker 1969); 3) Equating $\left\langle z^{2}\right\rangle^{1 / 2}$ with $z_{D}$ gives only a very rough estimate of the distance along the mean field over which a field line wanders to the surface of the disk.

The treatment of Jones (1.971 a) attempts to overcome these difficulties. He imagines an essentially two-dimensional situation in which the initial state of the magnetic field consists of a uniform field along the spiral arm embedded 
in a quiescent gas. Turbulent motion of the gas then deforms the lines of force in the z-direction, randomizing the field. The displacement, $s(x)$, of a field line from its initial position is assumed to be a random function of position along the mean field, expressible as a Fourier series in $\mathrm{x}$ with random phases. This implies that $s(x)$ is a homogeneous Gaussian process, i.e., that the multivariate probability density of $s$ or its derivatives at $\mathrm{N}$ (not necessarily distinct) points has the general form

$$
f(\vec{m})=(2 \pi)^{-N / 2}|M|^{-1 / 2} \exp \left[-\vec{m} \cdot M^{-1} \vec{m} / 2\right]
$$

where the components of the $\mathrm{N}$-tuple $\vec{m}$ are the values of $s$ or its derivatives at the $N$ points, and $M$ is the matrix whose elements are $\left\langle m_{i} m_{j}\right\rangle \cdot|M|$ is the determinant of $M$.

For a field line which passes through the origin, $x=z=0$, he uses eq. (4) to derive the probability density for displacement above or below the plane $z=0$ :

$$
f(z ; x) d z=\left\{2 \pi<s^{2}>[1-\psi(x)]\right\}^{-\frac{1}{2}} \exp \left\{-\frac{z^{2}}{2<s^{2}>[1-\psi(x)]}\right\} d z
$$

where $\psi(x)$ is the correlation furstion of the random process $s(x)^{1}$. Jones' model, as well as that of Jokipii and Parker, ignores gradients tr. $|\vec{B}|$ perpendicular to $\vec{B}_{0^{\circ}}$. This is, of course, insccurate, since the Galactic field is confined to the disk (Parker 1969). Note, however, that since $\psi(x) \rightarrow 0$ for large $x^{1}$, eq. (5) implies that in Jones' theory the r.m.s. displacement of a field line, $\left\langle s^{2}\right\rangle[1-\psi(x)]$, approaches a constant value as $x \rightarrow \infty$. Thus 
lines of force are confined to a bounded region in the z-direction, effectively simulating a dropoff in $|\vec{B}|$. The theory of Jokipii and Parker includes no such compensatory feature, as is evident from eq. (3).

jones goes on to derive an expression for the probability that a line of force passing through the origin crosses the level $z=z_{D}$ for the first time in the interval $(x, x+d x)$. Integrating this probability gives the probability that the line reaches $z=z_{D}$ in a distance along the mean field less than $x$. These probability densities are parametrized by the ratio $\delta=z_{D} / 2\left\langle s^{2}\right\rangle{ }^{1 / 2}$, which can be related to the correlation length and to the average angular deviation of lines of force from the direction of the mean field. For various possible values of $\delta$ Jones finds that the median value of $x / L$, i.e., the value for which the integrated probability of first crossing is $1 / 2$, is as given in Table 1. These results are in general agreement with those of Jokipii and Parker, who estimate that a field line has a $50 \%$ chance of reaching the surface of the disk in about two to six correlation lengths.

This brief outline makes it clear that Jones' theory has certain advantages over that of Jokipii and Parker. As already mentioned, the former model has a desirable asymptotic behavior for large values of $\mathrm{x}$. Furthermore, it makes no assumptions about the relative strengths of the mean and fluctuating fields. Finally, it yields a probability density, which enables one to make more precise statements about field line wandering than heretofore, and can be used, for instance, to investigate the statistical properties of cosmic ray parameters (Jones 1971 b). 
Essential to Jones' model, however, is the assumption that sometime in the past the field configuration was uniform along the spiral arm. Although this initial condition appears only indirectly in the probability density (5) [through $\left\langle s^{2}\right\rangle$ and $\psi(x)$, it is undesirable that the theory must rely on such an arbitrary and implausible hypothesis. The approach proposed in the next section avoids any mention of initial conditions, but retains the desirable features of Jones' theory, while incorporating the physical plausibility of the treatment of Jokipii and Parker. 
III. GAUSSIAN FIELD MODEL

The point of view to be suggested here retains the general description of the magnetic field embodied in eq. (1) ${ }^{2}$, with certain refinements concerning the fluctuating comprnent, $\vec{B}_{1}$.

1. $\vec{B}_{1}$ is assumed to have only a z-component, $\vec{B}_{1}=B_{1} \vec{I}_{z}$. Generalization to two components transverse to $\vec{B}_{0}$ is straightforward, but does not add significantly to the model; inclusion of a component parallel to $\vec{B}_{0}$ will be discussed in $\S V$. This assumption formalizes the approximation of Jokipii and Parker in which terms of order $\left|\vec{B}_{1}\right|^{2} / \mathrm{B}_{0}{ }^{2}$ are neglected. Note, however, that in the present case $\mathrm{B}_{1}{ }^{2} / \mathrm{B}_{\mathrm{O}}{ }^{2}$ is not necessarily small.

2. $B_{i}$ is assumed to be a function only of $x$. This is equivalent to neglecting gradients in $B_{1}$ transverse to $\vec{B}_{0}$, as is implicitly done by Jokipii and Parker. Adoption of such a slab model is not essential and is not physically motivated; it's virtue is the mathematical simplicity it affords.

3. $B_{1}(x)$ is assumed to be a homogeneous Gaussian process. The assumption of Gaussian statistics is plausible physically, since the magnitude of $B_{1}$ at any given time is the sum of a long sequence of random changes.

4. The statistical properties of $B_{1}(x)$ are required to be such that the r.m.s. displacement of a line of force is bounded in the z-direction. The motivation for this requirement is to compensate for neglect of gradients in the field strength perpendicular to the galactic disk. 
Using eq. (4), then, one can write the probability distribution for $\mathrm{B}_{1}(\mathrm{x})$ as

$$
\mathrm{f}\left(\mathrm{B}_{1} ; \mathrm{x}\right) \mathrm{dB}_{1}=\left\{2 \pi<\mathrm{B}_{1}{ }^{2}>\right\}^{\frac{1}{2}} \exp \left\{-\frac{\mathrm{B}_{1}{ }^{2}}{2<\mathrm{B}_{1}{ }^{2}>}\right\} \mathrm{dB}_{1} .
$$

The slope of a field line is

$$
z^{\prime}(x)=\frac{d z(x)}{d x}=\frac{B_{1}(x)}{B_{0}}
$$

By combining eqs. (6) and (7) one obtains the probability density for $z^{\prime}$ :

$$
f\left(z^{\prime} ; x\right) d z^{\prime}=\left\{\pi<z^{\prime 2}>\right\}^{-\frac{1}{z}} \exp \left\{-\frac{z^{\prime 2}}{2<z^{\prime 2}>}\right\} d z^{\prime}
$$

Consider the field line passing through the origin. The equation of this line is

$$
z(x)=\int_{0}^{x} z^{\prime}(s) d s
$$

Doob (1942) has pointed out that the probability distribution of the integral of a Gaussian process is itself a Gaussian process as long as the variance of the integral, in the present case $\left\langle z(x)^{2}\right\rangle$, is positive. Using eq. (9), $\left\langle z(x)^{2}\right\rangle$ is just

$$
\begin{aligned}
\left\langle z(x)^{2}\right\rangle & =\left\langle\int_{0}^{\mathrm{x}} \mathrm{z}^{\prime}(s) \mathrm{d} s \int_{0}^{\mathrm{x}} \mathrm{z}^{\prime}\left(\mathrm{s}^{\prime}\right) \mathrm{d} s^{\prime}\right\rangle \\
& =\int_{0}^{\mathrm{x}} \mathrm{d} s \int_{0}^{\mathrm{x}} \mathrm{d} s^{\prime}\left\langle\mathrm{z}^{\prime}\left(s^{\prime} \mathrm{z}^{\prime}\left(\mathrm{s}^{\prime}\right)\right\rangle\right. \\
& =\sigma^{2} \int_{0}^{\mathrm{x}} \mathrm{ds} \int_{0}^{\mathrm{x}} \mathrm{d} s^{\prime} \phi\left(s^{\prime}-s\right),
\end{aligned}
$$


where $\sigma^{2} \equiv\left\langle z^{\prime 2}\right\rangle=\left\langle B_{1}{ }^{2}\right\rangle / B_{0}^{2}$, and $\phi$ is the correlation function of the process $z^{\prime}(x)$. Interchanging the order of integration, one obtains

$$
\begin{aligned}
\left\langle z(x)^{2}\right\rangle & =2 \sigma^{2} \int_{0}^{x}(x-s) \phi(s) d s \\
& \equiv \sigma^{2} n(x) .
\end{aligned}
$$

Therefore, $z(x)$ will be a Gaussian process as long as $\phi$ is chosen so as to satisfy the condition

$$
n(x) \geq 0
$$

The probability distribution for $z(x)$ is then given by

$$
f(z ; x) d z=\left\{2 \pi \sigma^{2} n(x)\right\}^{\frac{1}{2}} \exp \left\{-\frac{z^{2}}{2 \sigma^{2} n(x)}\right\} d z
$$

Following Jones (1971 a), one can now derive an expression for the probability that the field line crosses the level. $z=z_{D}$ with positive slope in the interval $(x, x+d x)$. If the corresponding probability density is denoted $p\left(x ; z_{D}\right)$, then (Jones 1971 a)

$$
p\left(x ; z_{D}\right) d x=d x \int_{0}^{x} z^{\prime} f\left(z D^{\prime} z^{\prime} ; x\right) d z^{\prime}
$$


where $f\left(z, z^{\prime} ; x\right)$ is the bivariate density for $\left\{z(x), z^{\prime}(x)\right\}$, given by eq. (4) with $\mathbb{N}=2, \overrightarrow{\mathrm{m}}=\left\{z, z^{\prime}\right\}$, and

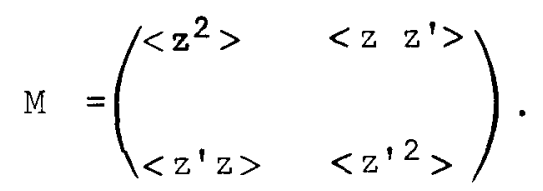

The cross correlation $\left\langle z(x) z^{\prime}(x)\right\rangle$ is

$$
\begin{aligned}
\left\langle z(x) z^{\prime}(z)\right\rangle & =\left\langle\int_{0}^{x} z^{\prime}(s) d s \cdot z^{\prime}(x)\right\rangle \\
& =\int_{0}^{x}\left\langle z^{\prime}(s) z^{\prime}(x)\right\rangle d s \\
& =\sigma^{2} \int_{0}^{x} \phi(s) d s \\
& \equiv \sigma^{2} \xi(x) .
\end{aligned}
$$

Using the definitions (11) and (15) one has

$$
|M|=\sigma^{4}\left(n-\xi^{2}\right)
$$

and

$$
M^{-1}=\frac{1}{\sigma^{2}\left(n-\xi^{2}\right)}\left(\begin{array}{cc}
1 & -\xi \\
-\xi & n
\end{array}\right) .
$$


Substituting these expressions in eq. (4),

$$
f\left(z, z^{\prime} ; x\right)=\left\{4 \pi^{2} \sigma^{4}\left(n-\xi^{2}\right)\right\}^{-\frac{1}{2}} \exp \left\{-\frac{z^{2}+n z^{\prime 2}-2 \xi z z^{\prime}}{2 \sigma^{2}\left(n-\xi^{2}\right)}\right\}
$$

$p\left(x ; z_{D}\right)$ is then obtained by inserting the right hand side of eq. (16) in eq. (14) and performing the integration. The result is

$$
\begin{aligned}
p\left(x ; z_{D}\right) & =\frac{\sqrt{n-\xi^{2}}}{2 \pi} \exp \left[-\frac{z_{D}^{2}}{2 \sigma^{2}\left(\eta-\xi^{2}\right)}\right] \\
& +\frac{z_{D} \xi}{2 \sigma_{0} \sqrt{2 \pi \eta^{3}}} \exp \left[-\frac{z_{D}^{2}}{2 \sigma^{2} \eta}\right]\left[1+\Phi\left(\frac{z_{D} \xi}{\sigma \sqrt{2 n\left(n-\xi^{2}\right)}}\right)\right]
\end{aligned}
$$

where $\Phi$ is the error function, $\Phi(s)=2 \pi^{-\frac{1}{2}} \int_{0}^{s} \exp \left(-t^{2}\right) d t$.

The probability that the line crosses the level $z=z_{D}$ for the first time in the interval $(\mathrm{x}, \mathrm{x}+\mathrm{dx}$ ) is (Jones 1971 a)

$$
\frac{d F\left(x ; z_{D}\right)}{d x} d x=p\left(x ; z_{D}\right) \exp \left\{-\int_{0}^{x} p\left(s ; z_{D}\right) d s\right\} d x
$$

and the integrated probability of first crossing somewhere between 0 and $x$ is

$$
F\left(x ; z_{D}\right)=1-\exp \left\{-\int_{0}^{x} p\left(s ; z_{D}\right) d s\right\}
$$


In order to evaluate these probabilities numerically, a form for the correlation function must be chosen. Even within the bounds of the mathematical constraints (see note 1 and in eq. (12) ) this choice is not entirely arbitrary, for the physical restriction that the r.m.s. displacement of a field line remain finite for large values of $x$ must also be satisfied (assumption 4). This requires that the integral

$$
\left\langle z(x)^{2}\right\rangle=2 \sigma^{2} \int_{0}^{x}(x-s) \phi(s) d s
$$

be bounded as $x \rightarrow \infty$ which, in turn, requires that $\phi$ be negative over part of its domain. Although this requirement clearly is not sufficient to determine $\phi$ uniquely, it severely restricts the possibilities. ${ }^{3}$ one function that meets all of the criteria is

$$
\phi(x)=\left(I-\frac{\pi x^{2}}{2 I^{2}}\right) \exp \left(-\frac{\pi x^{2}}{4 I^{2}}\right) .
$$

With this choice, the functions $n$ and $\xi$ [eqs. (11) and (15)] representing the mean square displacement of a field line and the slope-displacement correlation, respectively, are

$$
\begin{aligned}
& n(x)=\frac{4 L^{2}}{\pi}\left[1-\exp \left(-\frac{\pi x^{2}}{4 L^{2}}\right)\right] \\
& \xi(x)=x \exp \left(-\frac{\pi x^{2}}{4 L^{2}}\right)
\end{aligned}
$$


Figure 1 gives plots of $\phi, \eta, \xi$.

A simple physical interpretation of the form of the correlation function (20) can be obtained as follows. Consider the conditional probability density for the slope of a field line at a point $x$, given that the slope at $x=0$ has a particular value $z_{0}$. Using eq. (4) this is

$$
\begin{aligned}
f\left[z^{\prime}(x) \mid z_{0}^{\prime}\right] & =\frac{f\left[z^{\prime}(x), z^{\prime}(0)\right]}{f\left[z^{\prime}(0)\right]} \\
& =\left\{2 \pi \sigma^{2}[1-\phi(x)]^{2}\right\}^{-\frac{1}{2}} \exp \left\{-\frac{\left(z^{\prime}-\phi z_{0}^{\prime}\right)^{2}}{2 \sigma^{2}\left(1-\phi^{2}\right)}\right\},
\end{aligned}
$$

which is just a Gaussian of mean $z_{0} \phi(x)$. Thus, Figure 1 shows that within a region $0 \leqslant x \leqslant L$, the field line has an average slope of the same sign as $z_{0}$ ', reflecting the influence of the initial condition. For $x \gtrsim L$, on the other hand, the average slope has the opposite sign from $z_{0}^{\prime}$, which is necessary if the field line is to be confined to the Galactic disk.

In addition to providing a model that satisfies assumption 4, eq. (20) has the following advantage. When substituted in eq. (17) it allows $p\left(x ; z_{D}\right)$, $\mathrm{dF}\left(\mathrm{x} ; \mathrm{z}_{\mathrm{D}}\right) / \mathrm{dx}$ and $\mathrm{F}\left(\mathrm{x} ; \mathrm{z}_{\mathrm{D}}\right)$ to be expressed in terms of $\mathrm{x}$ and the single dimensionless number $\Delta \equiv \mathrm{z}_{D} / \sigma \mathrm{L}$. Then, by equating the mean square slope deviation in the present model with that in Jones' model, one obtains a relation between $\Delta$ and Jones' parameter $\delta$ :

$$
\Delta=\sqrt{\frac{8}{\pi}} \delta
$$


Using eq. (23) one finds that $\mathrm{p}(\mathrm{x} ; \Delta), \mathrm{dF}(\mathrm{x} ; \Delta) / \mathrm{dx}$ and $\mathrm{F}(\mathrm{x} ; \Delta)$ are identical to the corresponding expressions in Jones' theory. Thus, it is possible to reproduce the results of the latter theory without making any assumptions whatsoever about the initial condition of the magnetic field. 


\section{COMPARISON WITH OBSERVATIONS}

The range of the parameter $\Delta$ can be obtained from the values of $z_{D}, \sigma$ and $L$ inferred from observations. A summary of the available information is presented in Table 2. From this data one deduces that

$$
0.7<\Delta<1.9
$$

Eqs. (17) - (20) were used to obtain measures of the distance along the average field traversed by a field line between the origin and its point of first escape. In Figure 2 the most probable, median and mean values of this distance are plotted as functions of $\Delta$ in the range (24). The striking feature is the large differences among the different measures of the escape distance for a particular value of $\Delta$. Further illustration of the inherent indeterminacy of the escape distance is given in Figure 3, where the probability density for the escape point, $d F(x ; \Delta) / d x$, is plotted for $\Delta=1.3$.

It would appear that the escape distance of a field line is not a very useful concept. What is useful is the probability density $\mathrm{dF}(\mathrm{x} ; \Delta) / \mathrm{dx}$. It can, among other things, be used to investigate the effect of the stochastic magnetic field on the statistical properties of the propagation and confinement of cosmic rays in the Galaxy (Jones $1971 \mathrm{~b}$ ). 
V. SUMMARY AND DISCUSSION

Existing stochastic theories of the galactic magnetic field have been reviewed and used to formulate an eclectic model in an attempt to overcome the difficulties of the previous work. It has been found possible to reproduce the results of Jones' treatment (1971 a) while retaining the physical plausibility underlying the work of Jokipii and Parker (1969 a,b).

The new model shares a weakness common to both previous theories in that the longitudinal component of the fluctuating field has been neglected. That such fluctuations are not a priori negligible is clear, since the mechanism rardomizing the field is the motion of the interstellar gas, which certainly has components such as to generate fluctuations in the longitudinal direction. Furthermore, ignoring these fluctuations prevents the possibility of a field line doubling back on itself, and such reversals of direction of the field are observed to occur. It is not clear what effect inclusion of longitudinal fluctuations will have on the probability distribution $\mathrm{dF} / \mathrm{dx}$. Although such effects are not expected to change radically the nature of the results already obtained, the question is worthy of future investigation.

\section{ACKNOWLEDGEMENTS}

The author wishes to thank Dr. Frank Jones for suggesting the genera1 approach pursued in this paper as well as for helpful discussions during the course of the work. Useful conversations with Dr. Frank Kerr and Dr. Donat Wentzel are also appreciated. 
NOTES

1. A homogeneous process is one whose statistical properties are independent of position. For such a process, $\mathrm{g}(\mathrm{x})$,

$$
\left\langle\mathrm{g}(\mathrm{x})^{2}\right\rangle=\left\langle\mathrm{g}^{2}\right\rangle=\text { constant } .
$$

The two-point correlation function of $\mathrm{g}$ is translation invariant

$$
\left.<g(x) g\left(x^{\prime}\right)\right\rangle=\left\langle g^{2}\right\rangle \psi\left(\left|x^{\prime}-x\right|\right),
$$

and falls off rapidly for large values of $\left|x-x^{\prime}\right|$ :

$$
\left|\psi\left(\left|x^{\prime}-x\right|\right)\right| \ll 1, \quad\left|x-x^{\prime}\right| \gg I
$$

The length $\mathrm{L}$ is called the correlation length. (Yaglom 1962)

2. A fundamentally different model has been put forward by Mathewson (1968), who proposes a helical pattern for the lines of force. Recent observations by Manchester (1971), however, contradict this picture, and are, in fact, consistent with an average longitudinal field along the spiral arm.

3. Two correlation functions commonly used in stochastic process theory are the Gaussian, $\exp \left(-x^{2} / L^{2}\right)$, and the exponential, $\exp (-|x| / L)$. Since both of these functions are positive definite, neither can be used in the present application. 


\section{TABLE 1}

Median distance along the average field necessary for a field line to reach the surface of the Galactic disk for various values of $\delta(J$ ones 1971 a).

\begin{tabular}{cc}
\hline & $\mathrm{x} / \mathrm{L}$ \\
\hline 0.5 & 2.2 \\
0.8 & 4.3 \\
1.0 & 6.7 \\
\hline
\end{tabular}

\section{TABLE 2}

Values of the parameters $z_{D}, \sigma$ and L inferred from observations.

\begin{tabular}{|c|c|c|c|}
\hline Parameter & Minimum & Maximum & Reference \\
\hline$z_{D}(p c)$ & 100 & 200 & Schmidt (1956) \\
\hline$\sigma \quad(\operatorname{rad})$. & 0.7 & --- & Serkowski (1962) \\
\hline$L(p c)$ & 150 & & $\begin{array}{l}\text { Jokipii, Lerche \& } \\
\text { Schommer (1969) }\end{array}$ \\
\hline$L(p c)$ & & $2 z_{D}$ & Jokipii \& Lerche (1969) \\
\hline
\end{tabular}




\section{REFERENCES}

Doob, J.L. 1942, Ann. of Mathematics, 43, 351.

Fermi, E. 1949, Phys. Rev., 75, 1169.

Jokipii, J.R. and Lerche, I. 1969, Ap. J., 157, 1137.

Jokipii, J.R., Lerche, I. and Schommer, R.A. 1969, Ap. J. Lett., 157, L119.

Jokipii, J.R. and Parker, E.N. 1969a, Ap. J., 155, 777.

1969b, ibid., 799.

Jones, F.C. 1971a, Ap. J., 169, 477.

-_-_._. 1971b, Paper OG-111, 12th ICCR, Conference Papers

(Hobart: Univ, of Tasmania), $1,396$.

Manchester, R.N. 1971, Ap.J., 172, 43.

Mathewson, D.S. 1968, Ap. J., 153, L47.

Parker, E.N. 1970, in Interstellar Gas Dynamics (New York: Pergamon Press), pp. $168-183$.

Schmidt, M. 1956, B.A.N., 13, 247.

Serkowski, K. 1962, in Advances in Astronomy and Astrophysics, Vo1. 1

(New York: Academic Press), pp. 290-352.

Yaglom, A.M. 1962, An Introduction to the Theory of Stationary Random

Functions (New Jersey: Prentice Hall). 


\section{FIGURE CAPTIONS}

Figure 1. The functions $\phi, \eta$ and $\xi$ defined in Eqs. (20), (22) and (23).

Figure 2. Most probable, median and mean values of the distance along the average field traversed by a field line between the origin and its point of first escape as functions of $\Delta \equiv z_{D} / \sigma L$.

Figure 3. Probability density, $d F(x ; \Delta) / d x$, for first crossing when $\Delta \equiv z_{D} / \sigma L=1.3$ 

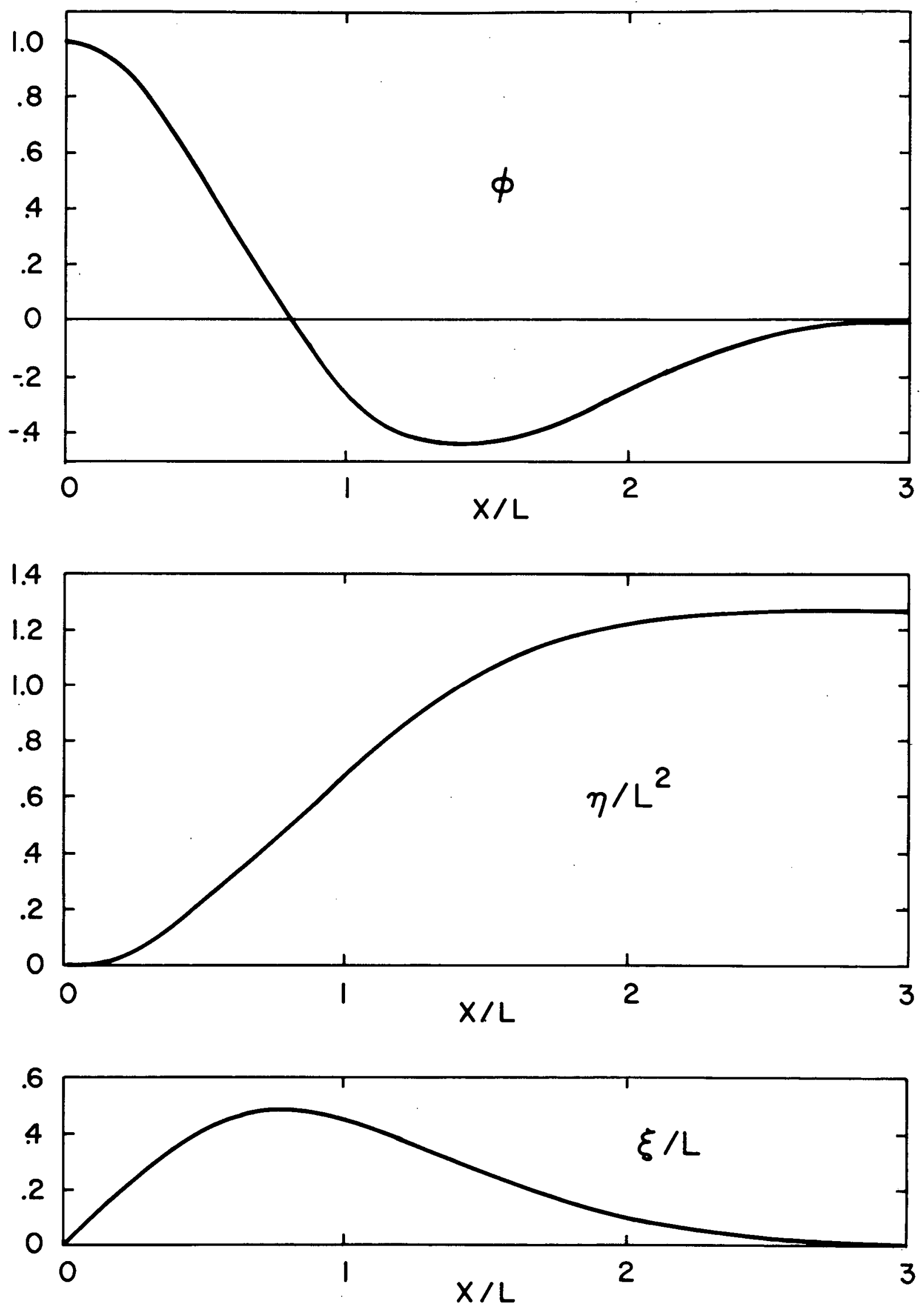

FIGURE 1 


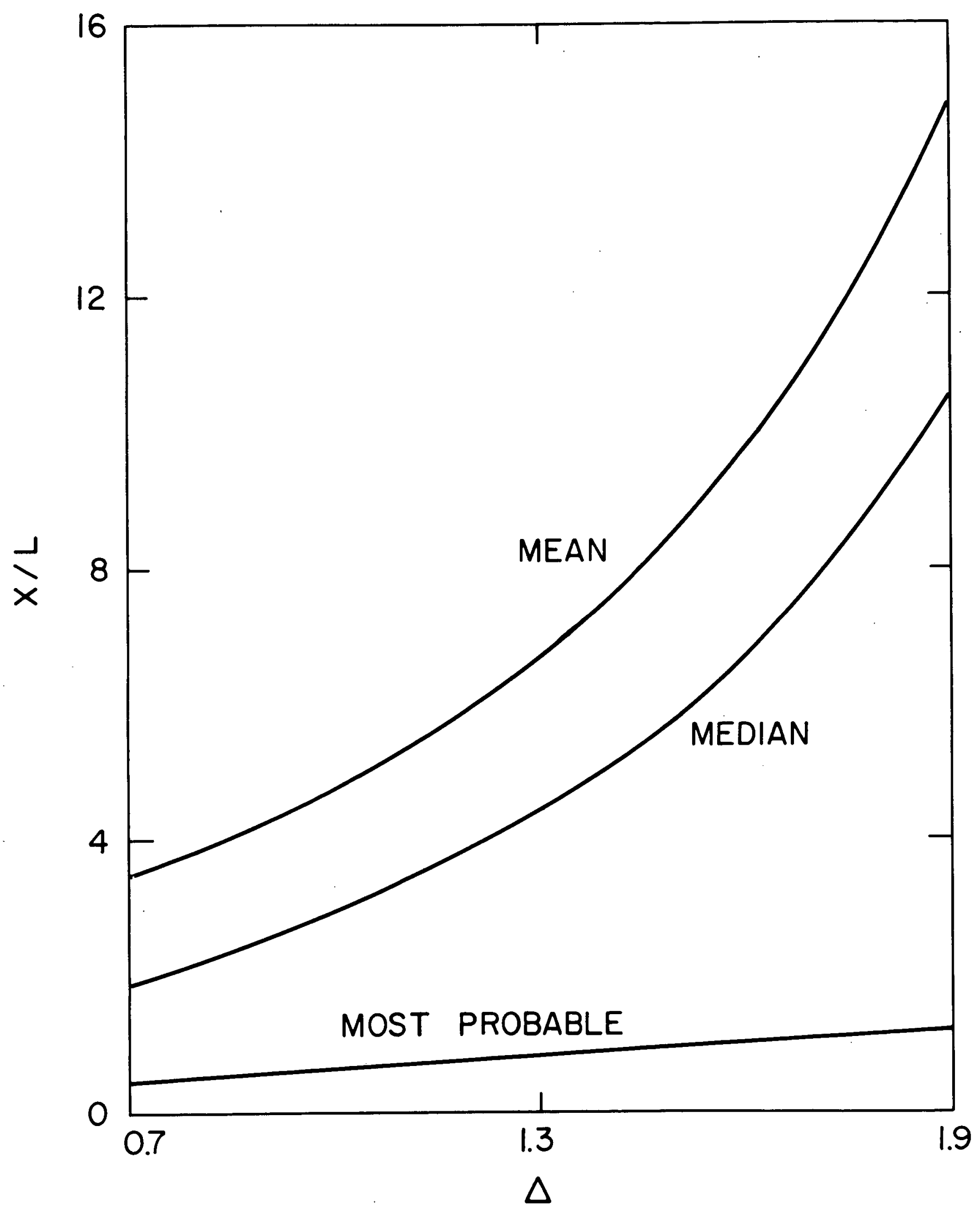

FIGURE 2 


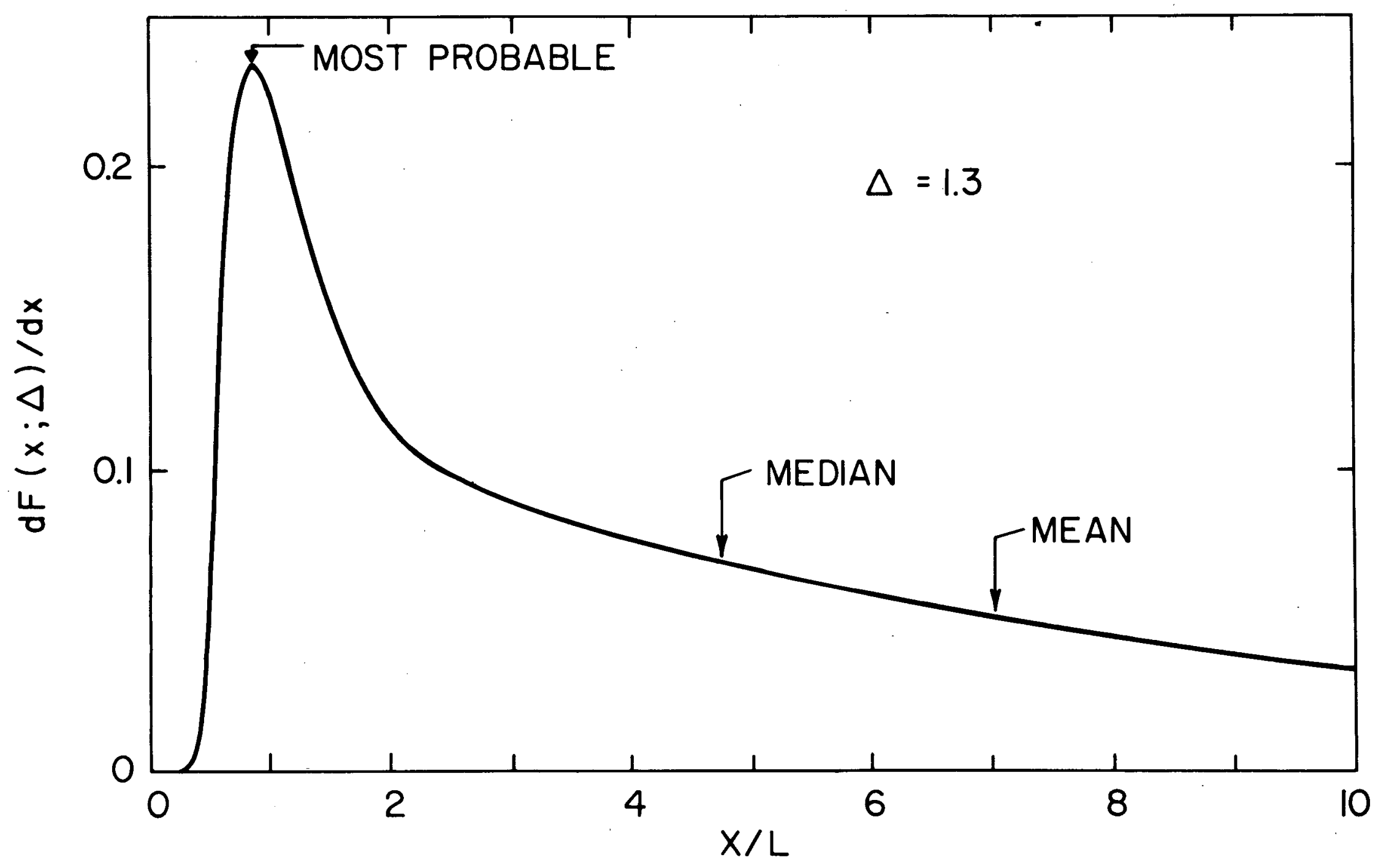

